Archives

$20 \mid 1998$

Miroirs de la Raison d'Etat

\title{
Autonomie et hétéronomie de la politique : la question de la finalité
}

Michel Sénellart

\section{OpenEdition}

Journals

Édition électronique

URL : http://journals.openedition.org/ccrh/2531

DOI : $10.4000 /$ ccrh.2531

ISSN : $1760-7906$

Éditeur

Centre de recherches historiques - EHESS

Édition imprimée

Date de publication : 12 avril 1998

ISSN : 0990-9141

Référence électronique

Michel Sénellart, « Autonomie et hétéronomie de la politique : la question de la finalité », Les Cahiers du Centre de Recherches Historiques [En ligne], 20 | 1998, mis en ligne le 20 avril 2009, consulté le 01 mai 2019. URL : http://journals.openedition.org/ccrh/2531; DOI : 10.4000/ccrh.2531

Ce document a été généré automatiquement le 1 mai 2019.

Article L.111-1 du Code de la propriété intellectuelle. 


\title{
Autonomie et hétéronomie de la politique : la question de la finalité
}

\author{
Michel Sénellart
}

1 Ce titre quelque peu abstrait renvoie tout d'abord «la question de la finalité » à un problème précis et capital traité par Marcel Gauchet dans son article ${ }^{1}$, mais aussi à une question, esquissée dans mon livre Les Arts de gouverner ${ }^{2}$ et qui fait l'objet de mes recherches actuelles: celle de l'autonomie et de l'hétéronomie de la politique, ou plus exactement celle du jeu entre autonomie et hétéronomie dans la formation du plan d'immanence de la politique depuis la fin du Moyen Âge. Après avoir résumé l'analyse de Marcel Gauchet, j'essaierai de préciser brièvement ce que j'entends par ces notions et m'appuierai sur l'exemple de Seckendorff - l'un des principaux théoriciens de l'« État de bien-être » (Wohlfahrtsstaat) au XVII ${ }^{\mathrm{e}}$ siècle - pour proposer une autre interprétation du rôle de la « finalité » dans la genèse de la raison politique moderne.

2 C'est dans la partie de son article intitulée « Raisonner l'État: la pensée comme défense » que Marcel Gauchet aborde la «question de la finalité ». Cette partie se divise en trois sections.

3 La première, p. 222-224 : sur la " posture défensive » des théoriciens de la raison d'État face à l'autonomisation du calcul politique, désignée sous le nom de «machiavélisme», posture décrite, à juste titre, comme la condition même de la théorisation de la raison d'État ${ }^{4}$. M. Gauchet cite deux exemples : celui de l'Italie, " épicentre du mouvement », et celui de l'Allemagne, "autre future terre classique de l'absence d'État-nation », l'un et l'autre témoignant d'une prégnance tardive de l'héritage aristotélicien.

4 La deuxième section, p. 224-232: sur le caractère confessionnellement neutre de la révolution religieuse et métaphysique de l'État, "révolution invisible» qui divise semblablement catholiques et protestants :

On ne peut aucunement identifier l'œuvre réactive des théoriciens de la raison

d'État à une cause religieuse strictement définie.

Thèse à nouveau soulignée à la fin de cette section, à propos de la théorie de «l'État de bien-être en Allemagne $»^{5}$. 
C'est ici que je voudrais soulever, sinon une objection, du moins une question. La doctrine sociale luthérienne - si elle a favorisé le souci du bien-être matériel des sujets, à des fins politiques de maintien de l'ordre et d'accroissement de la puissance - n'a-t-elle pas donné naissance à une tout autre conception de l'État, une toute autre logique de production du lien d'obéissance (ou du rapport de sujétion) que le discours catholique de la ContreRéforme, tel que l'a exposé Botero par exemple, dans une perspective classiquement mercantiliste? Aristotélisme et absence d'État suffisent-ils pour expliquer la genèse de «l'État de bien-être » en Allemagne? Une telle explication ne gomme-t-elle pas la spécificité de cette doctrine par rapport au discours italien de la ragion di Stato?

C'est dans cette section qu'est abordée «la question de la finalité ». J'y reviendrai plus bas, en précisant par là-même la question que je pose.

5 La troisième section, p. 232-234 : sur les divisions internes au discours de la raison d'État, à l'intérieur duquel Marcel Gauchet distingue deux grandes lignes stratégiques: une stratégie plutôt modératrice (Botero, Ammirato, Campanella, Boccalini); une stratégie plutôt conservatrice (Frachetta, Bonaventura, Palazzo, Zuccolo).

Bien que j'aie appelé « conservatrice », pour ma part ${ }^{6}$, la raison d'État que Marcel Gauchet qualifie de «modératrice", il n'y a pas là de désaccord. Voir le livre important de G. Borrelli, Ragion di Stato e Leviatano ${ }^{7}$, qui montre bien comment cet éventail de théories se déploie à l'intérieur d'un même paradigme conservateur. Il me parait plus difficile, en revanche, d'inscrire simplement Zuccolo - que Meinecke lui-même, peu tendre avec les publicistes antimachiavéliens, louait d'avoir écrit «sur la raison d'État l'ouvrage le plus bref, mais aussi le plus significatif » et, en qui Croce, non sans exagération, voyait «le plus profond philosophe politique de son temps ${ }^{8} »-$ dans la lignée des conservateurs. Mais l'important pour mon propos n'est pas là.

6 Revenons à la deuxième section de la partie de l'article qui m'intéresse ici. Le passage d'une économie du divin à une autre qui se joue, presque invisiblement, dans les nouvelles théories de la raison d'État, se vérifie, dit Marcel Gauchet, sur trois points fondamentaux :

7 a) le mode de liaison entre le politique et le religieux. Voir, p. 225, à propos du conflit entre deux visions du lien entre ici-bas et au-delà ;

8 b) la forme du corps politique. L'État, en naissant, subvertit la double articulation traditionnelle: forme corporative du lien social / insertion dans l'horizon de la communauté humaine universelle ;

9 c) l'inscription des fins dans l'ordre politique. Aussi congruente soit-elle avec la révolution scientifique (ruine du cosmos aristotélicien), la révolution intellectuelle,

[...] qui installe l'État en position suprême de principe d'ordre parmi les hommes est aussi une révolution intellectuelle, [trouve sa limite dans le fait qu'en politique], la question des fins [...] est inéliminable. Il ne saurait y avoir de pensée purement mécaniste du politique $»^{9}$.

[C'est pourquoi] tout l'effort des théoriciens de la raison d'État, en regard [de la mise entre parenthèses de la considération des fins, corrélative de l'émergence de l'État comme pure institution de puissance], va consister à rappeler l'État, justement, à ses raisons d'être [et à] ramener le bien commun au centre de [sa] définition ${ }^{10}$.

Les deux exemples cités sont ceux de Botero et de l'« État de bien-être » allemand. Je ne veux pas m'attarder ici sur Botero, partageant à peu près la lecture qu'en fait Marcel Gauchet. En revanche, je me demande si l'on peut interpréter de la même façon, comme 
une figure purement réactive face à l'avènement de l'État, la catégorie du Wohlfahrt dans la doctrine aristotélico-luthérienne de l'État autoritaire de bien-être.

Quelques mots pour préciser l'axe de mes recherches actuelles. Mon propos, dans Les Arts de gouverner, n'a pas été de faire l'archéologie conceptuelle de l'idée de raison d'État à travers les strates de la pensée médiévale, afin de retracer les voies souterraines conduisant à sa brusque émergence au XVI ${ }^{\mathrm{e}}$ siècle. Je ne suis pas sûr, en effet, qu'une telle entreprise ait un sens, l'idée de raison d'État étant liée au contexte d'une crise sans précédent, ce qui ne dispense pas, évidemment, d'en rechercher les prémisses, au Moyen Âge, dans le langage des politiques ou des juristes, comme l'a fait par exemple Gaines Post. Il n'a pas été, non plus, question de faire la généalogie de l'État moderne à partir des diverses pratiques de gouvernement - justice, guerre, fiscalité, police morale et sociale, diplomatie, ainsi que l'ensemble des fonctions symboliques assurées par le roi, tant au plan politique que religieux - qui se sont mises en place, codifiées, renforcées jusqu'à l'époque classique.

11 Mon intention était autre. Non pas rechercher les sources lointaines de l'idée de raison d'État, ni reconstituer les stratégies par lesquelles s'est construite, en Occident, l'institution étatique, mais essayer de repérer le moment où l'« art de gouverner », dans la culture chrétienne médiévale, commence à se dégager de la prudence royale traditionnelle, le moment, en d'autres termes, où se dessine le partage entre une conception éthico-religieuse et une conception technico-pratique de l'ars regiminis. Il m'a semblé en effet qu'il fallait situer avant Machiavel le moment où s'effectuent les transformations théoriques et pratiques qui ont rendu possible cette scission, déterminante pour la pensée politique moderne. Situer ce moment avant Machiavel ne signifie pas déplacer en amont la "rupture machiavélienne » comme si celle-ci était déjà accomplie avant qu'il ne lui donne, dans Le Prince, sa formulation fracassante. La "rupture machiavélienne", cela ne fait aucun doute, quand bien même on peut en trouver des signes précurseurs chez tel ou tel auteur, est inconcevable sans Machiavel, ou plutôt sans les conditions spécifiques de cet arte dello stato auquel, pendant quatorze ans, il a voué sa vie ${ }^{11}$. Toute la question, et c'est là où le schème de la rupture me paraît trop pauvre, trop globalement réducteur, est de savoir à quel niveau s'inscrit l'impact du langage machiavélien. Il importe, pour ce faire, de le replacer à l'intérieur d'une mutation plus profonde de la culture occidentale, celle par laquelle, dès le Moyen Âge, s'est constitué, au sein d'un ordre structuré par la transcendance, le plan d'immanence de la politique. Comment rendre compte de la genèse de ce dernier? On a tendance à confondre cette question avec celle de l'autonomie de la politique. Or je pense qu'elles sont bien distinctes, ou plutôt que la seconde ne se pose que dans le cadre défini par la première. Car le plan d'immanence de la politique, ce n'est pas seulement l'idée que la politique est autonome, fondatrice, instituante (Machiavel, Hobbes), mais aussi bien, celle qu'elle est tout à fait hétéronome, dépendante de variables objectives, constituée par les rapports mêmes des choses entre elles (Machiavel encore, Montesquieu, Marx). C'est ce jeu de l'autonomie et de l'hétéronomie, de la puissance absolue du prince et de la force contraignante des choses, qui fonde, me semble-t-il, le plan d'immanence de la politique. L'ambivalence de la raison d'État s'explique en grande partie par là. Mais la science de la " police », telle que la théorise par exemple Seckendorff, au XVII siècle, contre la ratio status absolutiste, ne peut-elle se comprendre comme l'effort de conjurer les périls de celle-ci, en redéfinissant, sur une base matérielle, descriptive et quantifiable, la radicale hétéronomie de la politique ? À travers ces questions je voudrais essayer de comprendre 
comment l'on est passé, non pas tant du gouvernement des hommes à l'administration des choses que du gouvernement par les hommes à l'administration selon les choses ${ }^{12}$.

Seckendorff est l'auteur du premier traité systématique sur la policey au XVII ${ }^{e}$ siècle $^{13}$, où il s'affirme comme un précurseur, sinon l'un des premiers représentants, de la conception de l'État de bien-être, Wohlfahrtsstaat, qui sera pleinement théorisée au XVIII siècle. La « police » désignant l'ensemble des moyens mis en œuvre dans l'administration de l'État pour assurer son bon ordre, le problème est de savoir dans quelle mesure elle s'inscrit dans le champ de problématisation de la raison d'État, prise au sens large; non pas la simple transgression violente des normes juridico-morales au nom de la nécessité, mais les diverses logiques étatiques servant à l'accroissement de la puissance. La question se pose, en effet, parce qu'à la différence de la France où la police s'inscrit dans une rationalité gouvernementale structurée par l'idée de raison d'État ${ }^{14}$, c'est contre cette dernière - ou du moins contre son acception machiavélienne - que s'est constituée, en Allemagne, la doctrine de la policey.

La ratio status n'était pas assimilée, en Allemagne, à la seule habileté machiavélique, mais constituait l'indice, le repère, éventuellement le slogan de lignes d'affrontements multiples, traversant les différents camps politiques et religieux. Il y avait place, dans ce champ complexe, pour une ratio status légaliste, chrétienne et conservatrice. Or Seckendorff, qui n'ignorait pas l'ambivalence du concept, l'écarte sans plus de commentaire. Preuve, me semble-t-il, que la policey ne s'inscrit pas à ses yeux dans le langage de la politique moderne, mais dans les traditions spécifiques des "États" allemands. À l'État de la ratio status, figure despotique d'une domination absolue, Seckendorff oppose le modèle d'une autorité patriarcale, attentive au bien-être commun.

Grâce à Dieu, dans les territoires allemands, nous ne connaissons pas ce type de pouvoir exercé par un seul qui, se plaçant au-dessus de tous les autres, détient avec ou sans droit une puissance considérable dont il use à son propre intérêt et avantage, selon sa volonté et son plaisir, comme un maître à l'égard de ses serviteurs et servantes $[. . .]^{15}$.

Au rebours d'un tel arbitraire,

[...] le gouvernement du prince territorial dans les principats et les territoires allemands, comme dans presque toute policey juste et bien ordonnée, n'est autre que la souveraineté suprême et éminente du prince territorial ou du seigneur qui gouverne droitement, utilisée et exercée par lui sur les «états» et les sujets du principat ainsi que sur le territoire lui-même et les choses qui en font partie, pour la conservation et l'affirmation de l'intérêt (Nutzen) et du bien-être communs, dans les affaires spirituelles et temporelles, et pour l'administration de la justice ${ }^{16}$.

Ce texte appelle trois remarques. La première sera brève. Les deux autres, en revanche, appelleront un plus long développement.

1. Seckendorff, tout d'abord, oppose la ratio status absolutiste à la finalité du gemeinen Nutzens und Wohlwesens, de l'utilité et du bien-être commun. Le gouvernement du bienêtre, prenant en charge l'ensemble des hommes et des choses, constitue donc l'alternative à la raison d'intérêt d'un pouvoir qui ne vise qu'à se renforcer lui-même.

16 2. À la différence de l'État despotique, qui traite ses sujets comme des esclaves, la finalité du Wohlfahrt implique la reconnaissance de leur liberté.

Puisque, comme nous l'avons rappelé, le pouvoir du seigneur territorial ne ressemble pas à la domination arbitraire d'un propriétaire sur ses domestiques, il est aisé de comprendre que les sujets dans le territoire ne sont pas des esclaves et n'appartiennent pas corps et biens à leur seigneur, mais doivent être dirigés et maintenus dans l'obéissance comme des êtres libres (freygeborne) et réunis sous un 
gouvernement juste pour le Wolhfahrt [au double sens, ici, du bien-être et du salut] de leur corps et de leur âme ${ }^{17}$.

L'affirmation de la liberté des sujets, sous la règle extérieure de contrainte d'une autorité garante du bien commun, s'articule chez Seckendorff à la critique d'une domination de type patrimonial, telle que l'exercent les $"$ Turcs et autres seigneuries barbares $»^{18}$. La théorie de la policey, ainsi, reconduit par certains aspects la conception médiévale du regimen - définition corporative de l'État, fonction directive du prince, double finalité temporelle et spirituelle-, mais l'inscrit dans une perspective anti-féodale. Le prince n'est pas le propriétaire des biens meubles et immeubles du territoire. C'est aux sujets qu'appartiennent leurs maisons, leurs champs, leurs vignes, leur argent, leur bétail, leur blé etc., et il ne peut en disposer à sa guise. Ce point est capital, car il permet à Seckendorff de faire entrer le jeu du marché dans l'économie dirigiste de la " police ». La liberté des sujets, en effet, ne réside pas dans la seule jouissance de leurs droits, naturels et coutumiers, mais dans la capacité de s'enrichir. Comme tous les mercantilistes, Seckendorff soutient qu'une population nombreuse fait la force de l'État. "L'abondance des sujets [...], [dit-il], est le véritable trésor des territoires $»^{19}$. Le prince a intérêt à favoriser son accroissement, pour augmenter les revenus de l'État, l'argent étant le nervus rerum gerendarum ${ }^{20}$. Il a également intérêt à ce que les sujets tirent profit de leurs biens, afin qu'ils lui obéissent plus volontiers.

Un bon et heureux gouvernement ne peut exister sans des "états " (Stände) valeureux, riches, obéissants et loyaux à l'égard de leur patrie. Il y a peu à attendre de sujets pauvres, pressurés, réduits à l'esclavage et la mendicité. Ils n'ont pas d'attachement pour leur prince. Ses succès et ses insuccès les laissent indifférents, parce qu'ils savent qu'ils restent misérables et que la dureté ou la cupidité du gouvernement ne leur permet pas d'améliorer leurs revenus ${ }^{21}$.

Mais, alors qu'en 1656, Seckendorff conçoit cette interaction d'intérêts dans le cadre d'une réglementation autoritaire, confiant à l'État le soin d'organiser l'activité économique, il en va tout autrement, quelques années plus tard, dans les remarques ajoutées à la troisième édition de son traité. À la suite d'un voyage en Hollande, effectué en 1657, constatant l'inefficacité des mesures de police pour assurer le développement économique du territoire, il préconise une plus grande liberté intérieure d'industrie et de commerce. Tandis que la Hollande attire des milliers d'Allemands, parce que tout homme valide, jeune ou vieux, peut y gagner quelque chose par son travail et que l'on y prend soin des pauvres et des malades, l'Allemagne, épuisée par la guerre, mais prisonnière aussi de structures archaïques, est incapable de pourvoir aux besoins du plus grand nombre.

On se plaint sans cesse du manque de travailleurs et du coût élevé des salaires (...), mais on ne tient pas compte du fait que ceux qui doivent chaque jour gagner leur vie ne trouvent pas assez de travail et s'en vont ailleurs ${ }^{22}$.

De là, pour stimuler l'emploi, toute une série de réformes nécessaires : élimination des corporations de métiers, allégement de la fiscalité, prise en charge des enfants de famille pauvre dans des établissements d'éducation, etc. Dans ce domaine, dit Seckendorff, der Eigennutz ist die Regul,

c'est l'intérêt particulier qui est la règle ${ }^{23}$.

La critique de la domination patrimoniale, à partir du principe de la liberté des sujets, ouvre donc l'espace du jeu des intérêts particuliers dans l'administration du bien-être commun, l'État ayant pour tâche, dans cette dynamique circulaire des intérêts, de désassujettir les hommes et non de renforcer indéfiniment son emprise sur eux. On ne saurait, bien sûr, qualifier Seckendorff de libéral. La sphère d'activité des individus reste 
déterminée par les impératifs du salut public. Mais il est important d'observer que la logique autoritaire de l'État de police, contrairement à l'image polémique et réductrice forgée, à la fin du XVIII ${ }^{\mathrm{e}}$ siècle, par les théoriciens libéraux, n'exclut pas entièrement celle du marché. Bien au contraire - et ce sera plus clair encore chez le principal théoricien de l'État de police, J. H. G. von Justi, un siècle plus tard -, elle crée les conditions, à certains égards, de sa mise en œuvre par la destruction des structures corporatives de la société d'ordres. La policey ne constitue pas une simple réaction conservatrice, face à l'essor d'une raison politique affranchie du cadre éthico-religieux traditionnel. Elle participe, sur un autre plan que la ratio status et selon d'autres modalités, au mouvement de rationalisation, voire, à certains égards, d'émancipation, des sociétés post-féodales.

3. Troisième remarque, enfin. En dépit du réalisme économique qui la caractérise, la conception du Wohlfahrt exposée par Seckendorff ne se ramène pas à un pur eudémonisme pragmatique. S'il en était ainsi, en dehors des techniques administratives propres à l'État de police, rien ne séparerait le gouvernement du bien-être de la raison d'intérêt. Or le Wohlfahrt, chez Seckendorff, doit s'entendre au double sens spirituel et temporel de «salut » et de "bonheur » ou «bien-être »-ambivalence qui me paraît tout à fait capitale pour l'interprétation du concept.

Le but du gouvernement du prince territorial réside dans la conservation et l'affirmation de l'intérêt et du bien-être (Wohlstand) communs dans les choses spirituelles et temporelles ${ }^{24}$.

Le gouvernement a affaire au spirituel, dans les États luthériens, depuis la paix d'Augsbourg (1555) qui marqua le transfert aux princes des attributions épiscopales où Seckendorff voit le retour au modèle du rex-sacerdos des premiers siècles ${ }^{25}$. Ce rôle est analysé dans les chapitres XIV à XVIII de la deuxième partie du traité. Il implique la nomination des dignitaires ecclésiastiques, l'établissement de consistoires, la convocation de synodes, l'entretien des églises, mais aussi, et surtout, la prise en charge des fonctions d'enseignement et d'assistance. L'école, l'hôpital et l'hospice relèvent désormais de la compétence du pouvoir princier. La première, en particulier, forme l'un des objets essentiels de la policey. La fin du gouvernement dans les choses temporelles, quant à elle, consiste en quatre points :

- la conservation par le prince du pouvoir et de la dignité attachés à sa position ;

- l'établissement de bonnes lois pour le maintien de la justice, de la paix et des ressources du peuple ;

- l'exercice de la juridiction suprême dans les conflits entre les sujets ;

- la défense du pouvoir, des lois et de la juridiction du prince contre les séditieux et les ennemis ${ }^{26}$.

Ces quatre points sont traités successivement par Seckendorff dans les chapitres VII à x de la même partie du livre. Le premier - la conservation de la majesté du prince-est développé, pour l'essentiel, sous la forme traditionnelle, mais renouvelée de façon originale, d'un "miroir du prince ». C'est dans le cadre du deuxième - la législation qu'intervient le concept précis de Wohlfahrt (à la différence du gemeine Wohlstand, ou bien commun). Comme on vient de le voir, les lois visent un triple but: paix, justice et Wohlfahrt ${ }^{27}$. Ce dernier consiste, avant tout, dans les deux biens précédents et, de façon plus particulière, dans le bon approvisionnement d'une population, l'accroissement de celle-ci et l'augmentation de ses richesses ${ }^{28}$. Le sens matériel du Wohlfahrt, ainsi, se dégage à peine de son sens juridico-moral. Il reste étroitement lié à l'idée médiévale de l'ordre juste, pax et justitia. Seckendorff le souligne sans ambiguïté : le but auquel concourent ces 
trois conditions est la saine conservation (die heilsame erhaltung) de la policey en hommage à Dieu - on retrouve ici l'idée de salus, dans ses connotations religieuses et séculières.

Seckendorff représente donc un moment de transition dans l'histoire de la policey. De la fin du Moyen Âge (le mot apparaît dans les dernières décennies $d u x^{e}$ siècle) au début du xvII ${ }^{e}$ siècle, la policey désigne soit le bon ordre d'une communauté, soit les lois assurant ce bon ordre. La policey, ainsi, se confond avec le règne du droit. Nulle différence entre la finalité du bien-être, ou bien commun (Wohlfahrt) et la justice. Le prince, chargé du soin de la policey, assure essentiellement un rôle de juge. À partir de la seconde moitié du XVII siècle, la policey tend à se dissocier de la justice, pour se transformer en une technologie de gestion des intérêts matériels de la société. Cette évolution, déjà repérable chez Seckendorff, dans les limites qu'on vient de voir, est liée à l'autonomisation du Wohlfahrt et à sa définition eudémoniste. Elle s'explique, en Allemagne, par un double contexte, doctrinal (l'implantation d'Aristote sur le terrain luthérien : articulation du bene beateque vivere et de l'officium, le Beruf assigné à l'obrigkeit) et historique (la reconstruction des États après la guerre de Trente ans). Avec le passage, alors, du modèle du prince-juge à celui du prince-législateur, la policey devient un instrument de renforcement de la puissance de l'État, par l'exploitation intensive de ses ressources humaines et matérielles. Cura promovendi salutem (Pütter) : elle a pour charge d'assurer corrélativement le bienêtre de chacun et le salut public. C'est sur ce plan, sans doute, qu'elle rejoint, par certains aspects, le gouvernement de la raison d'État. Elle possède néanmoins des traits spécifiques qui ne permettent pas de la rabattre sur ce dernier.

Seckendorff est un exemple particulièrement intéressant pour examiner les rapports entre ces deux logiques contemporaines, mais non homogènes l'une à l'autre, de la " police » et de la raison d'État. La manière dont il oppose le gouvernement du bien-être - i.e. le gute Regiment ordonné à la production du Wohlfahrt - aux calculs d'intérêt de la ratio status, s'inscrit encore en partie dans les catégories de la pensée médiévale. Mais elle $\mathrm{y}$ produit des effets de rupture, déterminants pour la constitution du domaine d'immanence de la politique moderne. Elle permet, en d'autres termes, de comprendre comment la modernité ne s'est pas formée en rupture avec la pensée religieuse, ni simplement dans l'axe d'une sécularisation progressive. Le religieux, du moins jusqu'au XVII ${ }^{e}$ siècle, n'est ni cette structure de pensée à laquelle la raison, d'un coup, aurait donné congé, ni celle qu'elle continuerait secrètement de reproduire à travers un langage laïcisé. Il me semble qu'il faut plutôt la concevoir comme un discours intervenant dans un certain jeu stratégique, ou, si l'on préfère, historico-politique, comme force de freinage, source de contestation, foyer d'accélération, levier de déplacement, point de bascule, etc. Le discours théorique et pratique de la policey - ses formes de rationalisation et ses modes d'instrumentation - offre sans doute, de ce point de vue, un champ d'analyse particulièrement fécond; la fin en fonction de laquelle il s'organise - le Wohlstand ou Wohlfahrt - se situant au carrefour de plusieurs axes sémantiques: le vocabulaire de l' utilitas, dont l'un des équivalents, dans le langage juridique romain, est le concept de salus (salus publica); le vocabulaire aristotélicien de l'eudaimonia ou du bene beateque vivere; le vocabulaire chrétien de la caritas, à travers les termes de Wohltat, Wohltätigkeit (bienfait, bienfaisance); et enfin celui du salut de l'âme. Ce sont ces différents axes qu'il faut articuler pour comprendre comment l'on est passé de la maxime cicéronienne Salus populi suprema lex à la définition de la policey, au XVIII ${ }^{\mathrm{e}}$ siècle, comme cura promovendi salutem, ou plutôt comment la première, qui traverse toute la littérature de la raison d'État et fait l'objet au XVII ${ }^{\mathrm{e}}$ siècle de multiples transpositions, chez Hobbes, par exemple, a pu se 
trouver reprise, intégrée, modifiée, recodifiée dans une économie du salus d'esprit religieux et eudémoniste.

\section{NOTES}

1. «L'État au miroir de la raison d'État », p. 228-232.

2. Paris, Seuil, « Des travaux », 1995.

3. Art. cit., p. 221-234.

4. Ibid., p. 224 : « La théorie de la raison d'État, c'est la pensée de l'État du dehors, là où il n'existe pas, à la lumière de ce que son affirmation effective tend à détruire ».

5. Ibid., p. $232:$ : [...] ici encore le critère de religion est de faible portée explicative, puisque, comme on sait, c'est dans l'Allemagne luthérienne que s'épanouira par la suite l'idée de «l'État de bien-être » - c'est-à-dire un État pourvu de raisons, le contre-type de l'État pour l'État de la raison d'État ».

6. Machiavélisme et raison d'État, Paris, PUF, « Philosophies », 1989.

7. Il Mulino, 1994 ; du même auteur, "Aristotélisme politique et raison d'État en Italie ", in Raison et déraison d'État, op. cit., p. 173-192.

8. Michel Sénellart, "Le problème de la raison d'État de Botero à Zuccolo (1589-1621) ", in Ch. Menasseyre et A. Tosel (eds), Figures italiennes de la rationalité, Kimé, 1997, p. 153-192.

9. Art. cit., p. 229.

10. Ibid., p. 231.

11. Lettre fameuse à $\mathrm{F}$. Vettori du 10 décembre 1513, in Lettres familières, traduction française de R. Barincou, Paris, Gallimard, t. II, p. 370.

12. Voir en particulier mon article «La Critique allemande de la raison d'État machiavélienne : Jacob Bornitz ", Corpus, 1997, p. 185-187, à propos de la sufficientia rerum.

13. Teutscher Fürsten-Stat, Francfort et Leipzig, 1656 (cité désormais TF, dans l'édition de 1665).

14. P. Napoli, «La conceptualisation d'un modèle juridico-politique sous l'Ancien Régime », Droits, 20, 1994, p. 186-191, qui met en évidence cet axe souveraineté - raison d'État - police.

15. TF, II, 1, p. 57-58.

16. Ibid., p. 58.

17. Ibid., II, 4, § 1, p. 78-79.

18. Ibid., III, 1, § 2, p. 364 .

19. Ibid., Additiones, $\S 42$, t. II, p. 183.

20. Ibid., Additiones, $\$ 37$, t. II, p. 145.

21. Ibid., Additiones, $\S 6$, t. II, p. 27.

22. Ibid., Additiones, $\S 42$, t. II, p. 185.

23. Ibid., p. 199.

24. Ibid., II, 1, § 6, p. 62.

25. Id., p. 63

26. Ibid., II, 1, § 8, p. 64.

27. Ibid., II, 8, p. 211.

28. Ces derniers points sont développés dans les § 3-4, p. 212-215. 